Klaus Rossenbeck

\title{
Zwei- und mehrsprachige Fachwörterbücher - Prolegomena zu Theorie und Praxis der Fachlexi- kographie*
}

O. In diesem Beitrag sollen — vor dem Hintergrund kritischer Auseinandersetzung mit Wörterbüchern, die vor allem das Sprachpaar Schwedisch und Deutsch betreffen - eine Reihe von zentralen Fragen der Fachlexikographie (FLG) ${ }^{1}$ diskutiert und, wo dies möglich ist, Ansätze zu ihrer Lösung in Theorie und Praxis aufgezeigt werden. Eine Aufarbeitung der gesamten metalexikographischen Forschung, die sich speziell oder am Rande mit fachlexikographischen Problemen beschäftigt, kann im Rahmen dieses Beitrags nicht geleistet werden, und das gleiche gilt für die Forschung zur allgemeinsprachlichen bilingualen Lexikographie, deren Problemformulierungen und Lösungsvorschläge im Hinblick auf ihre Übertragbarkeit auf die FLG zu untersuchen wären. Aus Platzgründen muß auch weitgehend darauf verzichtet werden, die hier aufgestellten Thesen und Postulate durch Beispielmaterial zu unterbauen. Dies alles muß einer geplanten Monographie über Theorie und Praxis der zwei- und der mehrsprachigen FLG vorbehalten bleiben.

Daß die hier erörterten Probleme zum großen Teil keineswegs neu sind, sondern daß sie die Lexikographen (die, wenn sie diesen Namen wirklich verdienen, immer auch, explizit oder implizit, Metalexikographen gewesen sind) praktisch seit eh und je beschäftigen, zeigt ein Blick in die Vorworte einer kleinen, recht beliebigen Sammlung älterer Wörterbücher, denen die über die einzelnen Abschnitte dieses Beitrags gesetzten Mottos entnommen sind. Auch wenn die Probleme alte Ahnen haben — niemand wird ernsthaft behaupten wollen, sie seien restlos geklärt, und ein weiteres "Nachdenken über Wörterbücher" sei damit überflüssig: die Diskussion über die theoretischen Probleme der fachsprachlichen Lexikographie ist eigentlich erst in letzter Zeit richtig in Gang gekommen!

\footnotetext{
* Diesem Beitrag liegen Vorträge zugrunde, die auf dem "Kopenhagener Werkstattgespräch" (1.2.6.1988; vgl. Kromann/Thomsen 1989) bzw. an der Wirtschaftsuniversität Århus (6.3.1990) gehalten wurden. Er ist eine Weiterentwicklung der hauptsächlich in Rossenbeck (1987) dargetanen Gedankengänge.

${ }^{1}$ Wenn im folgenden von Fachlexikographie (FLG) bzw. von Fachwörterbüchern (FWB) die Rede
} 


\section{Abgrenzung des Gegenstandes der FLG}

Något sådant [lexikon], [...], däri man på ett ögonblick kunde finna det man sökte, fanns absolut icke.

Bertels (1911, o.S.)

Die Tatsache, daß Benutzer von Fachwörterbüchern (FWB) oft nicht das finden, was sie suchen bzw. daß Material in den FWB verzeichnet ist, bei dem es sich zumindest trefflich darüber streiten ließe, ob es in ein FWB des betreffenden Fachgebietes aufgenommen werden sollte bzw. ob es überhaupt in ein FWB gehöre, hat seine Ursache sicher auch darin, daß zwei Probleme bis heute theoretisch wie praktisch nicht befriedigend gelöst sind: 1) Abgrenzung der Fachsprache(n) von der Allgemeinsprache; 2) Abgrenzung der Fächer untereinander und Zuordnung von Wortschatz zu einzelnen Fächern.

Von den in der Forschung zu diesen beiden Problemkomplexen aufgestellten Modellen ${ }^{2}$ könnten am ehesten diejenigen für eine Theorie der FLG nutzbar gemacht werden, bei denen der kommunikative Aspekt und in Verbindung damit das Konzept von "Zentrum und Peripherie" im Vordergrund stehen; aber gerade weil diese Modelle davon ausgehen, daß Allgemeinsprache und Fachsprache sich nicht entlang scharfer Trennungslinien voneinander scheiden lassen und auch die Grenzen zwischen den einzelnen Fächern durch zahlreiche Überschneidungen und diffuse Übergangszonen gekennzeichnet sind, haben sie m.E. noch keine untrüglichen und für den Lexikographen einfach zu handhabenden Kriterien erbracht, auf deren Grundlage eine eindeutige und unanfechtbare Wahl getroffen werden könnte, was in welche FWB aufzunehmen wäre und was dort als fachfremd beiseite gelassen werden sollte. Nicht gerade erleichtert wird die Abgrenzung durch die zunehmende Binnendifferenzierung der verschiedenen Fächer und die in immer stärkerem Maße wirksam werdenden interdisziplinären Ansätze, die die Grenzen zwischen den Fächern weiter verwischen.

Natürlich wird bei der Abgrenzung des Materials dem kommunikativen Aspekt entscheidendes Gewicht zukommen, was bedeutet, daß zunächst einmal die "fachlich/sachlichen Konstituenten eines Wirklichkeitsbe-

\footnotetext{
ist, so sind damit, soweit nichts anderes ausdrücklich vermerkt, die zweisprachige FLG und zweisprachige FWB gemeint.

${ }^{2} \mathrm{Zu}$ nennen wären hier vor allem zahlreiche Arbeiten von L. Hoffmann und H. Kalverkämper; eine ausführliche Auseinandersetzung mit diesen Arbeiten muß der geplanten Monographie über die Probleme der FLG vorbehalten bleiben.
} 
reichs, in dem sprachlich gehandelt wird"3, zu ermitteln sind, und daß im Anschluß daran die lexikographisch erfaßbaren sprachlichen Mittel, die der Kommunikation im betreffenden Wirklichkeitsbereich eigentümlich sind - neben streng definierten Termini also auch "Halbtermini" (Schmidt 1969, 446) und, mindestens ebenso wichtig: phraseologische Einheiten (s.u., Punkt 4) — registriert werden müssen. Bei der Entscheidung der Frage nach dem fachsprachlichen Charakter von Wortschatz wird es ebenso Grenzfälle geben wie bei der Zuordnung von Wortschatz zu einzelnen Fächern. Läßt sich dabei das subjektive Element gar nicht ganz ausschalten? Gibt es Kriterien, die mehr leisten als der gesunde Menschenverstand des Lexikographen, der sich leiten läßt von der gründlichen Kenntnis des Faches, das er bearbeitet, und von seiner auf langer Erfahrung basierenden engen Vertrautheit mit dem, was in der Kommunikation innerhalb seines Faches und über sein Fach von Bedeutung ist? Auf keinen Fall sollten die Grenzen so weit ausgedehnt werden, daß im fertigen FWB zwischen gewissen Teilen des aufgenommenen Materials und dem bearbeiteten Fach keinerlei Verbindung mehr erkennbar ist. ${ }^{4}$

\section{Materialerfassung}

Therföre har man alt igenom brukat the nyaste och bästa, så wäl Tyska som Swänska, fast med andra tungomål förknippade Lexica, af hwilka man utdraget thet nödigaste, och infördt $i$ thetta wärket [...].

Lind (1749, o.S.)

Alla Inhemska Lexikografers arbeten af något egentligt wärde, äfwensom de bästa utländska författares, hafwa blifwit samwetsgrannt jemförda; likwäl hafwa här influtit många ord, som i de förra blifwit förbigångna, tilläfwentyrs af förment brist på klassisk wärdighet, men hwilka dock för sitt dagliga bruk $i$ allmänna lifwet befunnits oumbärliga i ett mer omfattande Lexikon.

Öhrlander/Leffler (1852, o.S.)

Det säger sig själft, att jag måst anlita en del hjälpkällor, och jag tror mig hafva lyckats synnerligen väl $i$ valet af dessa. [...] För öfrigt har jag, som varande gammal köpman och korrespondent, hämtat mycket ur egen fatabur.

Bertels (1911, o.S.)

\footnotetext{
3 Buhlmann (1989, 101); wie facettenreich ein solcher "Wirklichkeitsbereich" sein kann, zeigt Buhlmann $(1989,102)$ am Beispiel Betriebswirtschaft, wo die Aufzählung der Teildisziplinen und Handlungsbereiche eine ganze Druckseite einnimmt.

${ }^{4}$ Vgl. die diesbezügliche Kritik an Parsenow (1975/1985) in Rossenbeck (1977, 80f.) und Rossenbeck $(1989,235 f)$.
} 
Daß einsprachige Wörterbücher und Fachlexika ein für die FLG unentbehrliches Hilfsmittel darstellen, ist nicht zu bezweifeln. Durch die Auswertung von Fachlexika läßt sich bereits ein erheblicher Teil der Lemmata in der Ausgangssprache erfassen, und manches auf dem Markt befindliche FWB hätte durch eine intensivere Nutzung dieses Mittels auffällige Lücken im Stichwortbestand schließen können. Die Materialerfassung kann sich aber nicht in einer Auswertung von Fachlexika in dem Sinne erschöpfen, daß einfach sämtliche Stichwörter, die darin zu belegen sind, unterschiedslos übernommen werden: einerseits sind nicht alle Stichwörter eines enzyklopädisch angelegten Lexikons auch gleichermaßen für Wörterbücher mit vordringlich sprachlichen Zielen von Interesse, und andererseits sind gewisse Ausdrücke, deren Aufnahme in letztere als wünschenswert erscheint, in ersteren überhaupt nicht zu belegen, oder sie treten wenigstens nicht als Stichwörter auf, sondern verbergen sich irgendwo in den enzyklopädischen Artikeln.

Überhaupt reichen einsprachige Wörterbücher und Fachlexika allein als Materialgrundlage für die FLG nicht aus: mancherlei kontrastiv relevantes Material ist in ihnen nicht zu finden. Dies gilt u.a. für das Gebiet der Ableitungen und der Komposita (s.u., Punkt 3) und besonders auch für das der fachsprachlichen Phraseologie (s.u., Punkt 4). Ebenfalls nicht ausreichend ist die bloße Benutzung des ausgangssprachlichen Materials eines schon vorhandenen FWB für die Erstellung von FWB mit anderer Zielsprache, ein vor allem in der kommerziellen Verlagslexikographie bereits häufig geübtes oder zumindest angestrebtes Verfahren, das unkritisch angewandt gewiß auch überflüssiges — weil sprachpaarspezifisch uninteressantes - Material in die neuen FWB schwemmt, was jedoch außer Platzverschwendung weiter keinen Schaden anrichtet; gleichzeitig besteht jedoch das Risiko, daß anderes Material, das im Hinblick auf die Sprachpaare der neu zu erstellenden FWB durchaus von Belang wäre, unbeachtet bleibt, weil es in dem FWB, das als Ausgangsbasis gewählt worden ist, aus verschiedenen Gründen nicht erscheint.

Daher wird der Lexikograph, der ein kommunikativ wirklich brauchbares FWB erstellen will, nicht umhinkommen, aus Eigenem zu schöpfen, was allerdings nicht so zu verstehen ist, daß er bei der Ansetzung des Ausgangsmaterials und/oder bei der Wahl von zielsprachlichen Entsprechungen munter darauflos phantasieren und mit eigenen Erfindungen brillieren soll ${ }^{5}$; vielmehr geht es darum, dieses Material aus den Quellen zu erarbeiten.

5 Ein in dieser Hinsicht besonders abschreckendes Beispiel ist Parsenow (1975/1985); vgl. dazu Rossenbeck $(1977,85)$ und Rossenbeck $(1989,232 f$. und 238f.). 
Wie aber soll ein solches Quellenstudium betrieben werden? Sicher könnte die Auswertung eines Textkorpus Erhebliches zur Gewinnung ausgangssprachlichen Materials, besonders im Hinblick auf Phraseologisches, beitragen, aber das Korpus müßte dann so groß sein, daß eine Bearbeitung ohne Computerunterstützung die Kräfte nicht nur eines einzelnen Lexikographen, sondern auch die von Lexikographen-Teams übersteigen würde und auch schon aus Zeit- und Kostengründen kaum in Frage käme. Eine maschinelle Auswertung hingegen, die in dieser Hinsicht wirklich etwas erbrächte, dürfte mit großen Komplikationen verbunden sein, da z.B. die Wörter, die feste Kollokationen bilden, in fortlaufenden Texten nicht unbedingt in unmittelbarer Nachbarschaft zueinander stehen.

Die Bedeutung eines Korpus für die FLG könnte gegebenenfalls darin bestehen, daß es die Stellung gezielter Fragen erlaubt, aber dazu wäre wiederum ein Vorwissen erforderlich, was die Anfrage an ein Korpus zur bloßen (in ihrem Wert allerdings keineswegs zu unterschätzenden) Kontrollmaßnahme herabsinken ließe, ohne daß sich Vermutungen, für die sich im Korpus keine Bestätigung finden läßt, schon als 'falsifiziert' betrachtet werden könnten. Ähnliches gälte dann auch für die Auswertung eines zielsprachlichen Korpus, die unbedingt parallel abzulaufen hätte, der aber dann ebenfalls in erster Linie eine Kontrollfunktion in bezug auf gewisse vom Lexikographen gemachte Annahmen zufiele. Denn für die Erfüllung der Hauptaufgabe eines jeden Lexikographen, die Findung von zielsprachlichen Äquivalenten, ist auch im Falle der maschinellen Auswertung eines zielsprachlichen Korpus ein Vorwissen unentbehrlich, das auf maschinellem Wege nicht zu erwerben ist. Anders ausgedrückt: die Koppelung von ausgangssprachlichem Material und zielsprachlichen Entsprechungen muß, vorläufig wenigstens, vom menschlichen Gehirn bewältigt werden, so daß in vielen Fällen eine gezielte Suche, bei der ausgangs- und zielsprachliche - in ihrer Entstehung voneinander unabhängige - Texte verwandten Inhalts nebeneinandergehalten und dabei auf bestimmte sprachliche Erscheinungen hin abgefragt werden, als der gegen- über einer Korpusauswertung weniger aufwendige und bessere Ergebnisse versprechende Weg erscheint (vgl. Rossenbeck 1989a, 204f.).

\section{Makrostruktur: die Stichwortauswahl (Komposita, Mehr- worttermini, Neologismen, 'historischer' Wortschatz)}

Quant aux substantifs \& autres mots composés, dont il y a un si grand nombre dans la langue allemande, \& dont on forge encore tous les jours de nouveaux, ce n' est pas un de moindres embarras, que de faire un choix juste \& proportionné aux besoins d'une autre langue comme la françoise. Nous avons tâché de tenir un juste milieu, en n'en donnant ni trop ni trop peu. Nous avons sur-tout 
inséré ceux, qui se rendent en françois par un mot simple ou autre terme, qui leur est particuliérement affecté, p.e. Nahrungs=Saft, chyle; Nagel=Schmidt, cloutier; Nacht=Stunden=Zeiger, nocturlabe \&c. [...] Nous souhaitons que le Public soit content du choix, que nous avons fait de ces mot composés.

Nouveau Dictionnaire (1782, o.S.)

Man är [...] långt ifrån att påstå sig lemna något fullkomligt, som upptoge alla möjliga, så äldre som nyare ord. —Att ord således her och der felas, är en ofullkomlighet som åtföljer alla Lexiker, i synnerhet dem öfver lefvande språk, hvilka oupphörligen föråldras, danas och omskapas. — Aldramest eger denna anmärkning rum i afseende på det Tyska språket, der nya ord snart sagdt dagligen sammansättas.

Wikforss $(1804$, o.S.)

Att upptaga alla sammansättningar är icke möjligt, icke en gång i ett större Lexicon, mycket mindre i ett Hand=Lexicon; likwäl äro i detta så många anförda, att de öfriga kunna genom analogien $i$ det närmaste finnas.

Wahrman (1814, o.S.)

Jag arbetade först ut Manuscriptet efter Adelung (Grammatisch=kritisch Wörterbuch der hochdeutschen Mundart, Leipzig 1793-1801); men har under fortsättningen följt Campe (Wörterbuch der Deutschen Sprache, Braunschweig 1807-1811), i hwad han är ordrikare än den förre; likwäl, som jag wågar tro, med warsamhet och urskillning, emedan jag icke ansett nödwändigt at uptaga hans nästan otaliga sammansatta ord, hwilket skulle hafwa gjort boken mera diger, utan at den derföre blifwit mera lärorik; [...]

Deleen $(1836$, o.S.)

Af sammansättningar äro de wigtigaste intagna; de öfriga skola genom analogien utan swårighet härledas från stamordet. I denna punkt, likasom wid intagandet af främmande ord, har man warit willrådig om rätta medelwägen mellan alltför mycket och alltför litet, - en klippa, hwarpå man wid utarbetandet af en ordbok så lätt strandar. Huru wida Förf. häruti, så wäl som i hela detta företag, warit mer eller mindre lyckliga, underställes sakkunnigas competentare dom.

Öhrlander/Leffler (1852, o.S.)

Zu den am häufigsten genannten Mängeln von FWB gehört deren Unvollständigkeit (vgl. Neubert 1990, 67f.). Unter den Gründen für diese Unzulänglichkeit ist ein an sich trivialer, weil unvermeidlicher, nämlich der, daß "das FWB, so schnell seine Verfasser auch arbeiten mögen, seiner Zeit grundsätzlich hinterher [ist]" (Neubert 1990, 70). Für Lücken, die sich auf diese Weise erklären lassen, braucht sich kein Lexikograph zu entschuldigen - für die Qualität eines FWB ist vielmehr entscheidend, wie gering oder wie groß der Abstand ist zwischen ihm und seiner eigenen Zeit, nicht der des Benutzers. 
Aber ist Lückenlosigkeit in der Abdeckung des Wortschatzes eines Fachgebietes überhaupt $\mathrm{zu}$ erreichen, ja, ist an der Forderung nach Lükkenlosigkeit wenigstens als einem Ideal auch nur festzuhalten? Und wie wäre Lückenlosigkeit angesichts der unter Punkt 1 genannten Schwierigkeiten eigentlich zu definieren? Neubert $(1990,70)$ räumt dem Ziel der "Vollständigkeit in sachlicher Hinsicht" eine relativ geringe Priorität ein, und der von ihm offensichtlich propagierte (wenn auch von ihm nicht so bezeichnete) 'Mut zur Lücke' erstreckt sich sogar auf "wichtige zentrale Wörter/Begriffe", die seiner Ansicht nach fehlen dürfen, "wenn sie keine Übersetzungsschwierigkeiten bieten" — als Beispiel führt er in einer Fußnote das Stichwort Mathematik an, bei dem er sich fragt, ob es "in einem FWB der Mathematik je nachgeschlagen wird".

Neuberts Argumentation liegt offensichtlich die Einstellung zugrunde, es sei immer der Lexikograph, der zu entscheiden habe, was Übersetzungsschwierigkeiten bietet und was nicht. Ganz abgesehen von den Kalamitäten, denen sich ein Lexikograph bei der Grenzziehung zwischen notwendigen und entbehrlichen Ausdrücken aus dem zentralen lexikalischen Bestand einer Fachsprache unweigerlich ausgesetzt sähe, sollte die Entscheidung über Aufnahme bzw. Ausschluß von Wortschatz immer aus dem Blickwinkel möglichst vieler potentieller Benutzer getroffen werden. Gerade weil es "den Nutzer" von FWB nach Neuberts eigener Einsicht (Neubert 1990, 71) nicht gibt, hat ein FWB auch die Aufgabe, als Nachschlagewerk zu dienen, anhand dessen auch der weniger kompetente Nutzer Zweifel ausräumen kann, ob die in der Zielsprache zu wählende Entsprechung sich durch 'Regelmäßigkeit' auszeichnet oder nicht. Macht der Lexikograph die Entscheidung über Aufnahme oder Ausschluß von Stichwörtern (bzw. in die Mikrostruktur zu verweisenden Materials, s.u.) von seinem eigenen — sicher oftmals subjektiv geprägten — Urteil abhängig, was kontrastiv schwierig ist und was nicht, so legt er im Extremfall dem Benutzer den Schluß nahe, daß alles das, was er im FWB nicht findet, irgendwie 'regelrecht' sei. Diese Methode zwingt den Benutzer letztlich zu oft nicht ungefährlichen Schlüssen ex silentio.

Das Ziel der "Vollständigkeit" betrachtet Neubert als nachrangig gegenüber der Erfüllung einer anderen Forderung, nämlich: "Das Fachwörterbuch von heute muß das Übersetzen der Texte von morgen mit dem Wortgut von gestern ermöglichen", was bedeute, "daß der Fachlexikograph bestrebt sein muß, das Übersetzen neugebildeter Wörter zu erleichtern, indem er beispielsweise [?] produktive Elemente von Fachwörtern als solche beschreibt und Regeln für ihre Verwendung bei der Fachwort- 
bildung angibt, vielleicht in Form von Mustern" (Neubert 1990, 70). Aus Neuberts Darstellung geht nicht klar und eindeutig hervor, ob sich die zu leistende Hilfestellung ausschließlich auf Neuprägungen beziehen soll und damit doch ein gewisser Anspruch auf Vollständigkeit bei den bereits konventionalisierten Bildungen aufrechterhalten wird, oder ob der Lexikograph auch in bezug auf letztere von der Last des Prinzips der Vollständigkeit befreit werden soll.

Wie dem auch sei - hinter Neuberts Überlegungen zeichnet sich der alte Streit um die Frage ab, inwieweit Komposita und Ableitungen, wofern sie nur 'regelmäßig' gebildet sind, in ein- bzw. in zweisprachigen Wörterbüchern behandelt werden sollen. Für zweisprachige Wörterbücher im allgemeinen und für FWB im besonderen sollte entgegen Neuberts Auffassung an der Forderung nach größtmöglicher Vollständigkeit grundsätzlich festgehalten werden, weil bei konventionalisierten Komposita und Ableitungen die in der Zielsprache zu treffende Wahl der Komponenten und der Wortbildungsmittel bei weitem nicht immer von den in der Ausgangssprache herrschenden Verhältnissen aus getroffen werden kann. Da etymologisierendes Übersetzen, Glied-für-Glied-Übersetzungen von Komposita und Ableitungen sowie vom Übersetzer auf eigene Faust vorgenommene Analogiebildungen nicht selten zu falschen Ergebnissen führen, ist eine möglichst umfassende Berücksichtigung von Ableitungen und Komposita unumgänglich, und zwar auch in solchen Fällen, wo sich ihre Gesamtbedeutung aus der Bedeutung ihrer Komponenten erschließen läßt und auch keine kontrastive Schwierigkeit vorliegt, weil die Wortbildungsstruktur und die die Bezeichnungen bildenden Komponenten in Ausgangs- und Zielsprache miteinander korrespondieren. ${ }^{6}$

Das bedeutet jedoch keineswegs, daß Neuberts Plädoyer für eine Lemmatisierung produktiver Wortbildungselemente und die Beifügung entsprechender Muster hinfällig wäre, im Gegenteil! Allerdings genügt die bloße Lemmatisierung eines Wortbildungselements allein besonders dann nicht, wenn die bei den zielsprachlichen Entsprechungen auftretende Wortbildung sich nicht oder nur beschränkt in Regeln pressen läßt (vgl. dazu Rossenbeck 1980, 49 und Rossenbeck 1981). Doch produktive Wort-

\footnotetext{
6 Vgl. das Vorwort zur 1. Auflage (1964) von I. Gullbergs FWB, wo ebenfalls die Rolle des FWB als 'Kontrollinstanz' hervorgehoben wird und Richtlinien formuliert werden, die einen vernünftigen Mittelweg darstellen: "I fråga om svenska sammansatta ord har endast de viktigaste medtagits, särskilt om den engelska översättningen avviker från vad man normalt skulle väntat sig. Sammansättningar, om vilka översättarna ofta svävar i tvivelsmål, har nästan alltid upptagits även om de skulle vara analogt bildade i de båda språken. På det sättet kan översättaren i varje fall få bekräftelse på att hans term kan användas." (Gullberg 1977, X)
} 
bildungselemente werden bekanntlich nicht nur zur Bildung neuer Termini verwendet, sondern sie treten auch bei der okkasionellen Wortbildung in Erscheinung, die, wenn sie nicht ausschließlich individualsprachlich bedingt ist, häufig als Mittel zur Herstellung von Textkohärenz dient. Rein okkasionelle Bildungen sind natürlich für die Aufnahme als selbständige Stichwörter disqualifiziert, aber gerade in solchen Fällen suchen weniger erfahrene Übersetzer nicht selten Rat und Hilfe beim Wörterbuch. Auf diesem Felde haben die Lemmatisierung von Wortbildungselementen und die Angabe von Wortbildungsmustern ihre wahre Funktion. Dieses Verfahren setzt allerdings beim Benutzer voraus, daß er produktive Wortbildungselemente auch als solche erkennt. Von geringerer Bedeutung ist dies, wenn das zu behandelnde Element vorwiegend als Vorderglied auftritt, weil es dann in der alphabetischen Ordnung des Wörterbuchs erscheint; bei Suffixoiden oder Elementen, die in erster Linie als Grundwort von Komposita vorkommen, empfiehlt es sich, den Benutzer durch eine spezielle Liste darauf aufmerksam zu machen, welche dieser Elemente lemmatisiert sind.

Die Abgrenzung zwischen einerseits Wortbildungen, die als im Sprachgebrauch konventionalisiert angesehen werden können und denen womöglich ein eigener begrifflicher Status zukommt - Voraussetzungen, die erst eine Verzeichnung in einem Wörterbuch rechtfertigen -, und andererseits solchen, die diese Kriterien nicht erfüllen und deshalb auszuschließen sind, wird jedoch immer der embarras bzw. die Klippe bleiben, von denen schon die Lexikographen des 18. und des 19. Jh.s sprechen.

Im übrigen wäre zu fragen, ob Neubert mit dem Hinweis auf "zentrale Wörter/Begriffe" wie Mathematik in FWB dieses Faches nicht ein Scheinproblem aufbaut. Bei der von Wörterbuchbenutzern und -kritikern gegen manche FWB in puncto Unvollständigkeit gerichteten Kritik geht es gar nicht in erster Linie um solche Fälle wie den von Neubert genannten, sondern einerseits um Lücken, die auf mangelnde Systematik in der Behandlung einheitlicher Begriffssysteme zurückzuführen sind (vgl. dazu Rossenbeck 1977, 80 und Rossenbeck 1985, 149f.), andererseits um Bezeichnungen, die nachweislich schon in der Zeit vor der Drucklegung des jeweiligen FWB in der fachlichen Kommunikation eine bedeutende Rolle gespielt haben und deren zielsprachliche Entsprechung auch beim besten Willen nicht mit Hilfe auch noch so guter Anleitungen zur fachsprachlichen Wortbildungslehre zu erschließen sind.

Mehrworttermini haben in einem FWB ihren selbstverständlich gegebenen Platz, zumal sie nicht selten erhebliche kontrastive 
Schwierigkeiten bieten (Wiedergabe durch Einworttermini oder durch Mehrworttermini nicht voraussehbarer Struktur). Für die Fachsprachentheorie wie für die Theorie der FLG gleichermaßen interessant ist dabei das Problem der Abgrenzung zwischen Mehrworttermini und anderen phraseologischen Einheiten, weil von seiner Lösung die Beantwortung gewisser Fragen abhängt, die die makro- und die mikrostrukturelle Gestaltung von FWB betreffen (Aufnahme als eigene Lemmata oder Verweisung in die Mikrostruktur unter anderen Stichwörtern).

Ein Problem eigener Art stellen die Neologismen dar, und zwar insofern, als ihr Status schwer zu bestimmen ist und sich bei der Erarbeitung eines FWB nur schwer voraussagen läßt, welche Neologismen sich über einen längeren Zeitraum im Sprachgebrauch halten werden. Die lexikologische Forschung hat bisher noch keine Ergebnisse erbracht, die eine im Hinblick auf lexikographische Arbeit einfache Operationalisierung des Begriffes Neologismus erlaubten. Als Grundsatz dürfte gelten, daß die Aufnahme kurzlebiger Neologismen eher zu tolerieren ist als der Ausschluß solcher, die sich dann doch als überlebensfähig erweisen, ohne daß nun auch die letzte Eintagsfliege registriert werden müßte. Bleibt auch hier nur der gesunde Menschenverstand des Lexikographen als letzte Instanz?

Die Probleme, die es bei der Behandlung von Neologismen zu bedenken gilt, stellen sich auch beim sog. 'historischen' Wortschatz, nur mit umgekehrtem Vorzeichen: Wie weit soll ein FWB in die Vergangenheit zurückgreifen, was soll z.B. bei der Neubearbeitung eines FWB erhalten bleiben, was soll ausgeschieden werden? "Was übrigens veraltetes Fachwortgut anbelangt, so ist offenbar der Grundsatz weithin stillschweigend anerkannt, daß ein FWB für ein bestimmtes Fachgebiet nicht auch gleichzeitig ein FWB für die Geschichte dieses Fachgebiets ist. Es gilt also nur das Wortgut der fachlichen Gegenwart als aufnahmewürdig." (Neubert 1990, 72) Eine strikte Befolgung dieses Grundsatzes würde jedoch zweifellos zu fragwürdigen Ergebnissen führen, wie Neubert selbst durch den in einer Fußnote gegebenen Hinweis auf eine Befragung, die unter Chemikern im Zusammenhang mit den Vorarbeiten für die Neubearbeitung eines chemischen Wörterbuchs veranstaltet wurde, zu erkennen gibt: die Befragten hätten sich gegen eine Eliminierung von - im Hinblick auf die IUPAC-Nomenklaturregeln - "vorreformatorischem" Wortschatz ausgesprochen, weil noch viele ältere Patente übersetzt würden.

Dieser Hinweis Neuberts zeigt, daß zur "fachlichen Gegenwart" aus 
sprachlichem Gesichtswinkel mehr gehört als eine streng synchronisch konzipierte Momentaufnahme, die den im Augenblick des Redaktionsschlusses des FWB gültigen Sprachzustand, soweit er durch Normen geregelt ist, repräsentiert. Anhand von Beispielen läßt sich außerdem leicht zeigen, welchen Abgrenzungsproblemen sich ein Fachlexikograph gegenübersähe, wenn er die "fachliche Gegenwart" im engen Sinne zum alleinigen Maßstab für Aufnahme oder Ausscheidung von ausgangssprachlichem Wortgut machte. Rigide angewandt würde dieses Prinzip bedeuten, daß nicht nur ältere Bezeichnungen für Begriffe, die z.B. aufgrund von Gesetzesänderungen etc. eine neue Bezeichnung erhalten haben, gestrichen werden müßten, sondern es wären auch jedesmal, wenn aus dem gleichen Grunde Sachen, Begriffe etc. aus der Realität verschwinden, die entsprechenden Bezeichnungen sofort auszusortieren: aus einem Fachwörterbuch der Wirtschaft, das als Bezugsrahmen die in der Bundesrepublik Deutschland herrschenden Verhältnisse zugrunde legt, müßte also Grundkapital spätestens 1988 verschwunden und durch gezeichnetes Kapital ersetzt sein, weil die seither gültigen Bilanzierungsvorschriften für Aktiengesellschaften diese neue Bezeichnung vorschreiben, und mit der Abschaffung der Börsenumsatzsteuer 1991 brauchte dieses Wort in einem FWB mit diesem Erscheinungsjahr auch als Lemma nicht mehr aufzutreten - die Widersinnigkeit eines solchen Verfahrens läge offen zu Tage: z.B. hat sich bis jetzt in der Wirtschaftsberichterstattung der Tagespresse gezeichnetes Kapital noch keineswegs als alleinige Bezeichnung gegenüber Grundkapital durchgesetzt. — Umgekehrt scheint es jedoch nicht erforderlich, in ein schwedisch-deutsches Fachwörterbuch für Recht und Wirtschaft noch 1985 Einträge wie "lägersmål (hist) außerehelicher Geschlechtsverkehr" oder "lönskaläge (hist) Geschlechtsverkehr zwischen unverheirateten Personen, außerehelicher Beischlaf” (Parsenow 1985) aufzunehmen, Begriffe, die sich auf Tatbestände beziehen, deren Strafbarkeit schon 1918 aufgehoben wurde. ${ }^{7}$

Aber das Problem der Neologismen hat auch eine von Neubert gar nicht diskutierte 'Kehrseite': wie steht es mit der Berücksichtigung 'historischen' Wortschatzes auf der zielsprachlichen Seite von Einträgen?

\footnotetext{
${ }^{7}$ Vgl. das Lemma lönskaläge in SAOB, Bd. 16 (1942). — Die angenehm undogmatischen Grundsätze, nach denen I. Gullberg in seinem FWB 'historischen' Wortschatz behandelt hat, dürften für die FLG wegweisend sein: "Även om det moderna ordförrådet utgör den viktigaste delen av det i denna ordbok redovisade materialet, har även många viktiga tidigare använda beteckningar upptagits, eftersom dylika termer i historiska återblickar ofta kan förorsaka översättarna stora svårigheter. Vid sådana termer har (hist.) eller (tidig.) oftast utsatts, om detta varit tekniskt möjligt." (Gullberg 1977, X).
} 
Sollte z.B. aktiekapital seit 1988 ausschließlich durch 'gezeichnetes Kapital' wiedergegeben werden, oder sollte daneben auch noch das offiziell ausgemusterte 'Grundkapital' aufgeführt werden? Sicher wäre es - aus Gründen, die den in Neuberts Fußnote zitierten vergleichbar sind, von Nachteil, wenn in einem FWB die Verbindungen zur sprachlichen Vergangenheit, besonders zur jüngsten, radikal gekappt würden. Die Forderung nach Synchronie sollte also auch hier nicht allzu eng ausgelegt werden, aber Abgrenzungsprobleme sind auch hier unvermeidlich: eine bestimmte Jahreszahl z.B., die einfach eine für alle Fälle geltende rückwärtige Grenze des zu berücksichtigenden Zeitraums bezeichnete, läßt sich nicht angeben, sondern Überlegungen, die die Gegebenheiten und Notwendigkeiten des Einzelfalles abwägen, werden den Ausschlag geben müssen. Wenn jedoch auf der zielsprachlichen Seite eine Angabe nicht dem neuesten Sprachgebrauch entspricht, ist selbstverständlich eine geeignete Markierung anzubringen, die auch mit Kommentaren, z.B. zum Verhältnis zwischen der neuen und der älteren Bezeichnung, versehen sein kann.

Gibt es auch in bezug auf Neologismen und 'historischen' Wortschatz keine andere Instanz als das gesunde Urteilsvermögen des Lexikographen, der kraft gründlicher Kenntnis der Kommunikation im jeweiligen Fach in der Lage ist zu entscheiden, was im weiteren Sinne zur sprachlichen Gegenwart eines Faches gehört und was nicht?

\section{Mikrostruktur: Fachsprachliche Phraseologie}

Til at förstå et språk är det icke nog at weta alla deß ord, man måste äfwen känna hwarje förändring af deß betydelse, hwilken uti sammansättningar och hela talesätt ofta är helt åtskillig. Jag har därföre bifogat en erforderlig Phraseologie för at wisa, $i$ hwilket särskilt förstånd man kan fatta och bruka wißa Tyska ord, som för öfrigt helt och hållet swara emot andra i Swenskan.

Möller (1782, o.S.)

Utgifvaren var i början sinnad, att uti detta Lexikon, äfven upptaga fraser; men sedan han funnit, att det derigenom skulle komma att blifva alltför vidlyftigt [...], har han, för att ej förfela ändamålet, varit nödsakad, att utesluta dem. Han förmodar, att detta så mycket heldre torde ursäktas, som de i de flesta Lexika upptagne fraser äro så påtaglige, att, då man känner bemärkelsen af de ord, som utgöra frasen, man ej lärer hafva någon svårighet att taga reda på sjelfva meningen däraf. Man nekar likväl icke, att talesätt förekomma, hvilka äro så egna, att de, utan något vidfogadt exempel, ej lätteligen kunna begripas; men dessa äro så sällsynta, att de snart sagdt alldrig förekomma, utom i den låga, dagliga, vulgära och provinsionella stilen, hvilken mycket sällan eger rum $i$ skrifter, för hvilkas explikation Lexiker egentligen äro skrifna. 
Wikforss (1804, o.S.)

[...] ordens särskilda betydelser har jag sökt att redigt utmärka; äfwensom fraseologien är betydlig, särdeles hwad egentliga Svecismer angår, hwilka alltid äro de swåraste att rätt uttrycka på andra språk.

Wahrman (1814, o.S.)

Ordens flerfaldiga betydelser, deras olikheter $i$ construction och anwändande samt andra särskilda egenheter äro dels hwar för sig, dels $i$ den hela arbetet genomgående utförliga fraseologien noggrannt upptagna [...].

Öhrlander/Leffler (1852, o.S.)

FWB müssen dem Gebiet der fachspezifischen und der fachbezogenen Phraseologie größte Aufmerksamkeit schenken, weil diese neben der Terminologie zu den zentralen Mitteln fachsprachlicher Kommunikation zählt. Zu berücksichtigen ist wirklich relevantes Material, d.h. solches, bei dem es sich nicht um beliebige freie Fügungen handelt, sondern solches, dem der Status einer phraseologischen Einheit (Fachwendung) wirklich zukommt $^{8}$; Vorrang ist dabei solchen Fällen einzuräumen, in denen kontrastive Schwierigkeiten auftreten. An anderer Stelle (Rossenbeck 1989a) wurde bereits dargelegt, worin die Bedeutung fachsprachlicher Phraseologie für die fachliche Kommunikation liegt und welche Schwierigkeiten sich bei der lexikographischen Bearbeitung dieses Gebiets ergeben: letztere bestehen darin, daß Fachwendungen und fachspezifische Kollokationen aus monolingualer Sicht über die in ihnen allenfalls enthaltenen Fachtermini hinaus oft keine Verständnisschwierigkeiten bieten, weshalb sie als nicht weiter erklärungsbedürftig in Bedeutungswörterbüchern und einsprachigen FWB auch nicht unbedingt verzeichnet zu werden brauchen (vgl. hierzu Rossenbeck (1989a, 202f.). Als festgeprägte Einheiten treten sie oft auch erst bei der Kontrastierung mit anderen Sprachen deutlich hervor. Schlechte Übersetzungen sind oft auch an den Verstößen gegen den phraseologiebezogenen Sprachgebrauch zu erkennen; den FWB fällt deshalb auch die Aufgabe zu, solchen Verstößen vorzubeugen.

\section{Aufnahme enzyklopädischer Elemente, authentischer Bele- ge und den Wortgebrauch illustrierender Beispiele}

Påflera ställen har jag i Ordboken sökt införa curiosa ur Naturalhistorien; men glömt at wid ordet $W$ a c h t e l anföra den märkwärdigheten, at det war blott en enda gång, och det wid Bellmansfesten den 26 Juli 1829, som en waktel slog $i$

8 Vgl. die diesbezügliche Kritik an Parsenow (1985) in Rossenbeck (1989, 232). 
en lind: ty de uppehålla sig wanligast i åkerfåror; [...].

Deleen (1836, o.S.)

Där något ord ej kunnat återgifvas med ett svenskt ord, eller där jag eljest kunnat förmoda, att en förklaring skulle vara välkommen, har jag lämnat en sådan $[\ldots]$.

Bertels (1911, o.S.)

Die Frage, ob die Aufnahme enzyklopädischer Elemente ins zweisprachige FWB verwerflich, legitim oder gar wünschenswert sei, ist nach wie vor umstritten. Während in Rossenbeck (1987, 278f.) für eine Aufnahme plädiert wird, lehnt Neubert (1990, 71f.) dieses Verfahren weitgehend $a b$. Der springende Punkt, der die Auseinandersetzung um das Für und Wider enzyklopädischer Elemente im FWB bestimmt, sind dabei die Annahmen, die über den Grad der bei den Benutzern von FWB vorhandenen fachlichen und fachsprachlichen Kompetenz gemacht werden.

In dieser Beziehung ist Rossenbeck (1987, 276f.) schon aus rein praktischen Gründen (Unmöglichkeit, besonders bei Beteiligung von 'kleineren' Sprachen, für jedes Fach eine Vielzahl von FWB zu erstellen - aktive und passive Wörterbücher für Benutzer unterschiedlicher Muttersprache, darüber hinaus solche, die auf die divergenten fachlichen und fachsprachlichen Kompetenzen potentieller Benutzergruppen - Fachleute, professionelle Übersetzer, Fachstudenten usw. - Rücksicht nehmen) geneigt, bei seiner Entscheidung für enzyklopädische Elemente und den Überlegungen, wie diese zu gestalten seien, von einer Art kleinstem gemeinsamem Nenner, der möglichst viele Benutzer umfaßt, auszugehen. Neubert (1990, 71f.) hingegen meint, der Lexikograph dürfe "dem Nutzer ein höheres Maß an fachlichem Mitdenkvermögen zutrauen, als die [?] gegenwärtigen FWB widerspiegeln"; er erläutert dies an einem Beispiel aus der Fachsprache der Pneumatik und fügt hinzu, daß ein Nutzer, der nicht in der Lage sei, die das Beispiel betreffenden Bezeichnungsdifferenzierungen, die zwar im Englischen, nicht jedoch im Deutschen vorhanden seien, vorzunehmen, "überhaupt kein Recht [hat], ein FWB zu benutzen!" (Neubert 1990, 72).

Auch hier scheint Neubert ausschließlich aus dem Blickwinkel des Lexikographen zu urteilen, der sogar Vorschriften erlassen möchte, wer das von ihm produzierte FWB benutzen darf und wer nicht. Da aber Wörterbuchbenutzer sich erfahrungsgemäß um solche Gebote oder Verbote wenig scheren, bleibt dem Lexikographen eigentlich nur der Weg, Unheil zu verhüten, indem er sein eigenes Wissen dem Benutzer nicht vorenthält, sondern diesem Brücken zum richtigen Sprachgebrauch baut. Am 
ehesten scheint dies möglich durch eine Ergänzung der rein sprachlichen Angaben durch enzyklopädisches Material, wie sie bereits in Rossenbeck (1987, 278-280) skizziert worden ist: Angabe von Definitionen auf ausgangs- wie auf zielsprachlicher Seite; kommentierende Zusätze z.B. bei Begriffsinkongruenzen; Aufnahme authentischer Belege und Beispiele. Neuerdings wird ein solches Verfahren auch von Kromann als Möglichkeit der FWB-Gestaltung akzeptiert: in Anlehnung an Wiegands Typologie einsprachiger FWB (Wiegand 1988) bezeichnet er den hier gemeinten Typ von FWB als "zweisprachiges fachliches Allbuch", dessen "genuiner Zweck" darin bestehe, "daß ein potentieller Benutzer aus den lexikographischen Daten Informationen sowohl zur Fachsprache als auch zur Sache in den parallelen Fachgebieten der fremd- bzw. muttersprachigen Fachwelt gewinnen kann" (Kromann/Thomsen 1989, 153).

In einem FWB, das letztlich sprachliche Ziele verfolgt, kann es jedoch nicht darum gehen, die einsprachigen Fachlexika aus beiden Sprachen je für sich voll zu ersetzen, sondern es sollte — um dem Benutzer eine schnelle und sichere Kontrolle des Grades an Äquivalenz zwischen Lemma und angebotenen Entsprechungen zu erlauben - 'komparatistisch' angelegt sein. Eine solche Kontrolle der Übereinstimmung von Begriffsmerkmalen muß sonst der sprachlich und fachlich nicht voll informierte FWB-Benutzer (wer sonst benutzt überhaupt ein FWB?) durch den Gang zu einsprachigen Nachschlagewerken und anderen Quellen (Lehrbüchern etc.) selbst leisten, um eine maximale Konformität des Informationsgehaltes von Ausgangstext und Übersetzung herzustellen. Der inhaltliche Vergleich enzyklopädischer Quellen zu bestimmten Erscheinungen der ausgangs- und der zielsprachigen Fachwelt ist ein meist mühsamer und zeitraubender Arbeitsgang, der dem FWB-Benutzer durch gezielte Angaben abgenommen werden kann. In den "enzyklopädischen Glossen" (Kromann/Riiber/Rosbach 1984, 196) und den dazu eventuell nötigen Kommentaren sollte daher das Hauptgewicht auf die kontrastiven Schwierigkeiten gelegt werden, d.h. Dinge wie Begriffsinkongruenzen in Ausgangs- und Zielsprache, auf kritische Punkte, die sich aus der unterschiedlichen Stellung eines Begriffs in ausgangs- und zielsprachigem Begriffssystem ergeben, auf das Verhältnis zwischen ausgangs- und zielsprachlichen Bezeichnungen z.B. bei 1:2-, 1:3-, 2:3-Entsprechungen, auf eventuelle Abweichungen in bezug auf die stilistische Ebene etc. Dagegen kann bei voller inhaltlicher Übereinstimmung die Glossierung knapper gefaßt werden.

Authentische Belege und Beispiele sollten in keinem FWB fehlen; sie 
sollten nach Möglichkeit sowohl auf der ausgangssprachlichen wie auf der zielsprachlichen Seite angebracht und dabei so gewählt werden, daß sie im Idealfall folgende Aufgaben erfüllen können: 1) sie beweisen, daß es sich bei den im FWB-Eintrag gemachten Angaben nicht um Phantasiebildungen des Lexikographen, sondern um den in der fachsprachlichen Kommunikation konventionalisierten Sprachgebrauch handelt; 2) sie können enzyklopädische Glossen ersetzen, wenn sie selbst eine Definition oder sonst eine geeignete Erklärung enthalten; 3) sie können durch Wahl geeigneter Kontexte die Äquivalenzbeziehungen zwischen ausgangssprachlichen und zielsprachlichen Bezeichnungen beleuchten; 4) sie können die Verwendung von Ausdrücken illustrieren, z.B. ihr Vorkommen in Kollokationen (vgl. Rossenbeck (1987, 280). Am Prinzip der Authentizität sollte auf keinen Fall gerüttelt werden; zu diskutieren wäre allenfalls eine Abmilderung dieses Prinzips im Sinne einer die Quellenauszüge verkürzenden Bearbeitung des Belegmaterials: eine solche wäre eventuell im Interesse der Platzersparnis zu vertreten. Die Bildung eigener Beispiele durch den Lexikographen wird sich trotzdem nicht immer ganz vermeiden lassen, doch sollten auch sie eine Stütze in authentischem Material haben. ${ }^{9}$

Weil das Durcharbeiten der enzyklopädischen Glossen, der Kommentare und der Belege Zeit erfordert, wird sich die "Benutzerzeit" derartig strukturierter FWB-Artikel zwar gegenüber solchen, die sich mit der unkommentierten Angabe von 1:1-Entsprechungen begnügen, gewiß verlängern. Aber die "Zeit des Benutzers" wird damit dennoch geschont, weil er im Endeffekt einen Zeitgewinn dadurch erzielen dürfte, daß ihm die sonst oft notwendigen Nachforschungen in verschiedenen einsprachigen Quellen erspart bleiben, und eine moderne drucktechnische Gestaltung des FWB, die einen leichten Überblick über die Einträge ermöglicht, kann ein übriges tun, die "Benutzerzeit" in erträglichen Grenzen zu halten.

\section{Grammatik im Fachwörterbuch}

Det allrasvåraste vid Tyska språkets skrifvande och talande är utan tvifvel att veta, när ett Substantiv har Vokalförändring, som blifvit utmärkt genom en *, och till hvad deklination det hörer, som i denna bok är bestämdt genom ziffrorna 1. 2. 3. 4. 5. 6., samt om ett neutralt Verb konjugeras med haben eller seyn, hvilket äfven blifvit uppgifvit, ehuruväl allt endast med afseende på min egen Grammatika [...], och är man fast öfvertygad, att ingen är $i$ stånd att skrifva Tyska språket felfritt med tillhjelp av Lexicer, som icke lemna någon upplysning

\footnotetext{
${ }^{9}$ Bei Sarcevic' $(1988,309)$ findet sich der Hinweis auf ein mir bis dahin unbekanntes und bis heute unzugängliches FWB (Dictionnaire juridique 1978), in dem das Prinzip der Angabe von Originalbe-
} 
om allt detta; helst som Grammatikan, utom en evinnerlig sammangyttring af reglor, som genast upphäfvas af tusende undantag, icke härutinnan kan lemna tillräckligt biträde.

Heinrich (1828:V)

Grammatische Angaben sollten auch im zweisprachigen FWB nicht fehlen — gemäß der obenerhobenen Forderung, daß sie für Benutzer beider Muttersprachen benutzbar sein sollten, sollten diese Angaben Ausgangs- und Zielsprache umfassen. Wichtig ist auch hier, daß für die fachsprachliche Kommunikation relevante Angaben gemacht werden und nicht der Platz auf Nebensächliches oder gar Belangloses verschwendet wird. Seit langem ist z.B. bekannt, daß gewisse Formen aus der Verbflexion (2. Person Sing. und Imperativ der 2. Person im Sing. und im Plur.) in den allermeisten Fachtextsorten so gut wie keine Rolle spielen, so daß bezweifelt werden muß, ob in einem Fachwörterbuch für Recht und Wirtschaft Einträge wie: "aufgeien du geitest auf, aufgegeit, geie auf!" oder "ausflaggen du flaggtest aus, ausgeflaggt, flagg aus!" (Parsenow 1975/1985) zu rechtfertigen sind.

\section{Wahl der Metasprache}

Nos devanciers n'avoient en vue que ce qui pouvoit être utile aux Allemands; c'est peutêtre la raison pourquoi ils ont souvent expliqué l'allemand par l'allemand même, leur travail devenoit par conséquent inutile aux François. Pour rendre donc à ces derniers par rapport à la langue allemande le même service qu' on avoit rendu aux Allemands par rapport à la langue françoise, nous avons fait de la partie allemande la premiere \& principale partie, \& nous avons donné aux mots allemands une explication en françois plus ample qu'on n'avoit jamais fait.

Nouveau Dictionnaire (1782:2v)

FWB brauchen m.E. in Wahl und Verwendung der Metasprache nicht die gleiche Strenge walten zu lassen wie allgemeine Wörterbücher. Bei den Informationen zur Grammatik, den Markierungen der Stilebene und ähnlichen Angaben, die ohnehin meist bloß die Form von Siglen haben, empfiehlt sich eine einheitliche Metasprache, während in den enzyklopädischen Zusätzen Ausgangs- wie Zielsprache als "Glossierungssprache" (Kromann/Riiber/Rosbach 1984, 167 und 193) zugelassen werden sollten. Das Abgehen von der Forderung nach ein-

legen sowohl auf der ausgangssprachlichen wie der zielsprachlichen Seite angewandt ist. — Lehnstrup (1989a) berichtet über die — offenbar von Rossenbeck (1987) unabhängige — "Erfindung" eines neuen ("otraditionell") Typs von FWB, der den in letzterer Arbeit erhobenen Forderungen weitestgehend entspricht und der an einem thematisch eng begrenzten Bereich auch bereits eine Verwirklichung in der Praxis erfahren hat (Lehnstrup 1989). 
heitlicher Metasprache in letzterem Falle erlaubt dem Lexikographen ein das Verfahren vereinfachendes Zitieren von Definitionen etc. aus einsprachigen Nachschlagewerken beider Sprachbereiche, und vom Benutzer eines FWB muß vorausgesetzt werden können, daß er der Ausgangssprache und der Zielsprache soweit mächtig ist, daß er sich in beiden Sprachen mit dem Inhalt enzyklopädischer Glossen ohne Schwierigkeiten vertraut machen kann: diese Fähigkeit würde ja auch sonst von ihm erwartet, wenn er sich auf eigene Faust kundig machen müßte.

\section{Zuverlässigkeit der Angaben im Fachwörterbuch}

Hwad man i främsta rummet fordrar af ett Lexikon är dess tillförlitlighet och dess motswarighet till det ändamål, som därmed warit åsyftadt. [...] Omtänksam försiktighet wid upptagandet och en noggrann pröfning wid behandlingen af hwarje ord hafwa icke ett ögonblick blifwit lemnade ur sigte.

Öhrlander/Leffler (1852, o.S.)

De stora allmänna ordböckerna bjuda på synnerligen litet $i$ affärsväg, och hvad som finnes, är svårt att uppleta och $i$ många fall felaktigt. [...] Hvad sägs t.ex. om sådana fel, delvis verkliga praktblommor, som att [...] "prestanda" (egentl. lat. praestanda = avgifter) [öfversättes] med "Priestergeist (= prästanda), [...].

Bertels (1911, o.S.)

Die von Öhrlander/Leffler erhobene Forderung nach Zuverlässigkeit ist heute so gültig wie vor beinahe 140 Jahren, und in besonderem Maße ist sie an FWB zu stellen, da falsche Angaben z.B. auf wirtschaftlichem oder juristischem Gebiet fatale Folgen haben können (vgl. Rossenbeck 1977, 84f.). Vom Ziel der Erfüllung dieser Forderung sind wir in der modernen schwedisch-deutschen und deutsch-schwedischen Lexikographie in einigen Fällen leider mehr, in anderen weniger weit entfernt. Bei anderen Sprachpaaren mag es Wörterbücher geben, die als rühmenswerte Gegenbeispiele herhalten können — doch die Klagen von Wörterbuchbenutzern und -kritikern lassen die Unzuverlässigkeit als weitverbreitetes Problem erscheinen. Bertels gibt leider nicht an, aus welchen "förut i bokmarknaden befintliga ordböcker" er zitiert; aber seine Zitate klingen, als seien sie allesamt direkt aus Prisma (1974/1980; vgl. dazu Rossenbeck 1975 und Rossenbeck 1981a) oder aus Parsenow (1975/1985) entnommen: ein prinzipieller Unterschied zwischen der Wiedergabe von prestanda durch 'Priestergeist' einerseits und des finanzwissenschaftlichen Ausdrucks Deckungsklausel ('Vorschrift, nach der in öffentlichen Haushalten sog. ordentliche Ausgaben durch Steuern etc., nicht durch Kredite zu finanzieren sind') durch 'betäckningsklausul' (betäckning 'Deckung' i.S.v. 'Besprin- 
gen, Beschälen') andererseits (Parsenow 1975/1985, wo betäckningsklausul schließlich zu allem Übel auch noch im schwedisch-deutschen Teil als Lemma auftaucht!), dürfte nur schwer auszumachen sein.

Gewiß läßt sich der Grad der Zuverlässigkeit der in einem Wörterbuch gemachten Angaben durch deren Verifizierung in anderen - primären oder sekundären - Quellen auf ein hohes Niveau bringen. Doch haben gegen dieses Prinzip der Verifizierung (in Öhrlander/Lefflers altertümlicher Sprache pröfning genannt), gewisse Lexikographen immer wieder nur allzu bedenkenlos verstoßen, indem sie nicht einmal von den im eigenen Vorwort genannten Nachschlagewerken angemessenen Gebrauch gemacht haben - anders sind viele fehlerhafte Angaben nicht zu erklären.

Beim Vorgang der Verifizierung sollten im Normalfall folgende Arbeitsschritte eingehalten werden: 1) Kontrolle, daß der Begriffsinhalt des ausgangssprachlichen Ausdrucks richtig erfaßt ist — in zweisprachigen Wörterbüchern anzutreffende Fehler lassen sich nicht selten aus die Ausgangssprache betreffenden Mißverständnissen erklären, und die obenvorgeschlagene Glossierung auch ausgangssprachlicher Termini bildete bereits auf dieser Stufe eine wirksame Sperre gegen fehlerhafte Einträge; 2) Kontrolle, daß der als zielsprachliche Entsprechung ins Auge gefaßte Ausdruck sich in der Zielsprache auch wirklich belegen läßt — primäres Ziel muß es immer sein, daß zur Wiedergabe konventionalisierter Fachausdrücke ebensolche in der Zielsprache verwendet werden und nicht etwa "Phantasiebildungen" oder "langatmige Umschreibungen mit gemeinsprachlichen Mitteln", vor denen schon Jumpelt (1954, 111) gewarnt hat; "[...] the lexicographer's task is to 'record', not to 'create' terminology [...]" (Sarcevic'1988, 312); 3) Kontrolle, daß begriffliche Übereinstimmung zwišchen ausgangssprachlicher und zielsprachlicher Bezeichnung vorliegt — auch hier bildet das Beibringen von enzyklopädischen Glossen eine wirksame Sperre gegen Fehler, und z.B. können auf diese Weise "falsche Freunde" (Wortpaare wie z.B. bolagsskatt / Gesellschaftsteuer oder medbestämmandelagen / Mitbestimmungsgesetz, die auch von Lexikographen irrtümlich zusammengekoppelt worden sind) relativ einfach aus dem FWB ferngehalten werden; 4) Kontrolle, daß zielsprachliche Ausdrücke, wenn sie denn auf unvermeidliche eigene Bildungen des Lexikographen zurückgehen, so gewählt sind, daß sie in den referentiellen Bezugsrahmen der Zielsprache hineinpassen und beim 'Empfänger' Assoziationen wecken, die ihn nicht in die Irre führen. Von

${ }^{10}$ Im Zusammenhang mit der Erörterung des Problems der Lemmatisierung von Wortbildungselementen als ein für den Benutzer gedachtes Hilfsmittel bei der Übersetzung neugebildeter Wörter schreibt Neubert: "Er [= der Fachlexikograph] muß überhaupt möglichst viele Fachwortüberset- 
eigenen Bildungen sollte der Lexikograph allerdings überaus sparsamen Gebrauch machen, prinzipiell eigentlich nur dann, wenn im Bezeichnungs- oder im Begriffssystem der Zielsprache echte Lücken vorliegen ${ }^{10}$; in solchen Fällen sollte der Benutzer auch unbedingt darauf aufmerksam gemacht werden, daß - und aus welchem Grunde — das FWB keinen konventionalisierten Fachausdruck anbieten kann. ${ }^{11}$

\section{Mehrsprachige Fachwörterbücher}

Die metalexikographische Forschung hat sich, soweit ich sehe, bisher des Problems der mehrsprachigen FWB nur in sehr geringem Umfang angenommen. Die Benutzerforschung hat $\mathrm{m} . \mathrm{W}$. auch noch nicht danach gefragt, wer auf welche Weise für welche Zwecke solche Wörterbücher benutzt. Von der Beantwortung dieser Fragen aber hängt die Anlage eines solchen FWB entscheidend ab. Folgende Modelle sind denkbar:

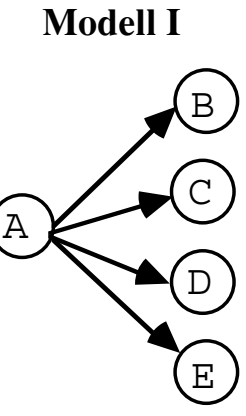

\section{Modell II}

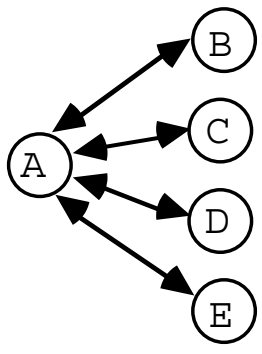

Modell III

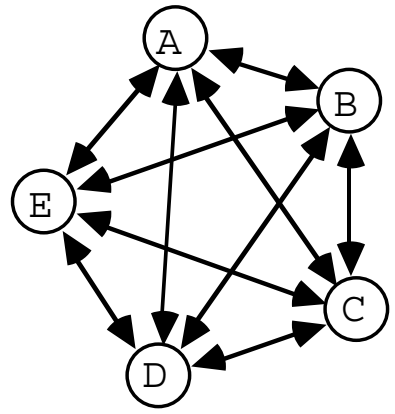

Modell I: Das Wörterbuch hat eine Ausgangssprache und mehrere Zielsprachen, d.h., es ist nur unidirektional verwendbar. - Modell II: Eine Ausgangssprache und je eine Zielsprache stehen gleichberechtigt nebeneinander; die über Register erschlossenen Einträge auf der

zungsprobleme voraussehen und ihre Lösung zu unterstützen suchen. Dabei ist es ihm [= dem Fachlexikographen!] nicht nur nicht verboten, selbst Wörter zu bilden, sondern er ist dazu schlechterdings verpflichtet [...]" (Neubert 1990, 70f.). Diese Formulierungen sind u.U. dazu geeignet, Mißverständnisse hervorzurufen, denn der Fachlexikograph kann für Wörter, die er noch gar nicht kennt, selbstverständlich keine eigenen Wortbildungen vorschlagen — dies muß der Übersetzer auf der Grundlage der ihm an die Hand gegebenen Muster selbst leisten. Neuberts Formulierung kann deshalb den Eindruck erwecken, daß er die Forderung erhebt, dem Fachlexikographen sei generell das Recht zuzubilligen, eigene Wortbildungen vorzunehmen. Eine solche Forderung wäre jedoch abzulehnen.

${ }^{11}$ Die wichtige Frage, inwieweit zweisprachige Wörterbücher im allgemeinen und FWB im besonderen in solchen Fällen eine normensetzende Funktion und eine normierende Wirkung haben, muß hier unerörtert bleiben. 
zielsprachlichen Seite dienen ihrerseits auch als ausgangssprachliches Material, so daß die Einträge bidirektional funktionieren. - Modell III: Alle berücksichtigten Sprachen sind füreinander zugleich Ausgangs- und Zielsprachen, jede kann auf jede, und zwar in beiden Richtungen, bezogen werden.

Es ist leicht einsehbar, daß bei Modell I noch am ehesten die Aussicht besteht, mit Erfolg Forderungen wie die nach Zuverlässigkeit der Einträge und maximaler inhaltlicher Kongruenz von ausgangs- und zielsprachlichen Einträgen sowie nach Einbeziehung von Phraseologie, authentischen Belegen oder enzyklopädischen Kommentaren zu verwirklichen, weil es sich im Grunde um verschiedene unidirektionale Wörterbücher handelt, die man genauso gut auch äußerlich als solche betrachten und behandeln könnte. Nicht zu übersehen ist, daß bei einem solchen FWB der ganze 'Apparat' erheblichen Umfang annehmen müßte.

Sinnvoll erscheinen solche mehrsprachigen FWB deshalb in erster Linie in solchen Fällen, wo die enzyklopädischen Glossen nur auf der ausgangssprachlichen Seite angebracht zu werden brauchten, weil auf der zielsprachlichen Seite per definitionem inhaltliche Konformität mit der ausgangssprachlichen besteht, d.h. also z.B. in FWB, die eine von einer Ausgangssprache ausgehende und auf verschiedene Zielsprachen wirkende Sprachnormierung entfalten sollen (verbindliche Regelung des Sprachgebrauchs in Übersetzungen aus der Ausgangssprache, z.B. in technischen Dokumenten eines multinationalen Unternehmens ohne einheitliche Konzernsprache): hier kann es von Vorteil sein, wenn Äquivalente aus verschiedenen Zielsprachen an einem Ort gesammelt sind.

Schon Modell II bringt sehr viel mehr und kaum noch zu bewältigende Probleme mit sich, weil Einträge in FWB oft aufgrund von begrifflichen Inkongruenzen nicht einfach umkehrbar sind. Besteht gar aus dem Blickwinkel einer Ausgangssprache eine begriffliche und/oder lexikalische Lücke im System einer Zielsprache, die mit Hilfe von Umschreibungen geschlossen werden muß, dann wird die Umkehrung noch problematischer, wenn nicht gänzlich sinnlos. Wenn ein Wörterbuch dieses Typs wirklich bidirektional funktionieren sollte, dann müßte solches Material praktisch ausgeschlossen werden, und da von solchen Fällen mal das eine, mal das andere Sprachpaar betroffen wäre, stellt sich die Frage, was dann von dem ganzen Wörterbuch eigentlich noch übrigbliebe.

Modell III erscheint als das komplizierteste, weil all die genannten Probleme sich darin potenzieren. Am ehesten scheint noch der Wortschatz im naturwissenschaftlichen oder im technischen Bereich, wo sich nach 
laienhafter Vorstellung meist mit 1:1-Entsprechungen (Schlagworte wären hier: Identität der Begriffe in verschiedenen Sprachgebieten aufgrund der Tatsache, daß sie kulturell ungebunden sind; internationale Normung von Bezeichnungen) und daher auch mit relativ problemlos umkehrbaren Wörterbucheinträgen arbeiten läßt, für eine lexikographische Darstellung, die dem Modell III entspricht, geeignet zu sein. Daß diese Vorstellung von begrifflichen und sprachlichen Verhältnissen in dem genannten Bereich oft nicht mit den Realitäten übereinstimmt, läßt sich deutlich an einem Beispiel ablesen, das in Neubert (1970) behandelt ist (engl. valve und die Vielzahl seiner deutschen Entsprechungen).

Trotz dieser erkennbaren Schwierigkeiten ist das multidirektionale Modell das explizit oder implizit ins Auge gefaßte Ideal mehrsprachiger FWB, denn die Erschließung aller zielsprachlichen Einträge durch Register soll ja im Prinzip den Zugriff zu allen anderen Sprachen des Wörterbuchs ermöglichen. Ein FWB, in dem fünf Sprachen berücksichtigt sind, soll praktisch zwanzig andere FWB ersetzen, und sind neun Sprachen einbezogen, eine in diesem Zusammenhang keineswegs ungewöhnliche Zahl, sind es deren gar 72. Die Schwierigkeiten, die damit verbunden sind, solche FWB zu erstellen und sie gemäß den obenaufgestellten Postulaten funktionstauglich zu machen, sind evident. An gewissen im Druck erschienenen Glossaren und FWB ließe sich beispielhaft nachweisen, wie sehr sie hinter ihren selbstgesteckten Zielen zurückbleiben. Mehrsprachige FWB dürften also grundsätzlich nur mit Vorsicht zu genießen sein! 


\section{Literatur}

\section{Zitierte Wörterbücher}

[Bertels, John (1911)], Bertels' Tyska Affärslexikon. Praktisk Handbok och Uppslagsbok för Tysk Handelskorrespondens. Svensk-Tyska. Tysk-Svenska. Göteborg

Deleen, Carl (1836), Tysk och Swensk Ordbok. Örebro

Dictionnaire juridique neerlandais-français avec vocabulaire français-neerlandais, droit privé. Amsterdam, Anvers 1978 (zitiert nach Sarcevic'(1988)

Gullberg, Ingvar E. (1977), Svensk-engelsk fackordb̌̌k. Stockholm, 2. Aufl.

Heinrich, Carl (1828), Nytt och fullständigt svenskt och tyskt lexicon, med alla sådana upplysningar, som Grammatikan ej så noga och bestämdt kan uppgifva. Stockholm

Lehnstrup, Rita (1989), Joint venture — engelske og danske termer. o.O.

Lind, Olof (1749), Teutsch-Schwedisches und Schwedisch-Teutsches Lexicon oder Wörter=Buch. Orda=Bok på Tyska och Swänska, så ock på Swänska och Tyska [...]. Stockholm

Möller, J. G. P. (1782), Teutsch=Schwedisches und Schwedisch=Teutsches Wörterbuch. Tysk och Swensk, samt Swensk och Tysk Ord=Bok. Första Delen. Greifswald

Nouveau dictionnaire allemand-françois et françois-allemand, à l'usage des deux nations. Tome I. Contenant l'allemand expliqué par le françois. Strasbourg, 3. Aufl. 1782

Öhrlander, C. N.; Leffler, O. E. (1852), Tetraglott-Lexikon för öfversättningar från svenskan till tyska, franska och engelska språken. Stockholm

Parsenow, Günter (1975/1985), Fachwörterbuch für Recht und Wirtschaft. Schwedisch/ Deutsch—Deutsch/Schwedisch. Köln usw., 1. Aufl. 1975, 2. neubearbeitete und erweiterte Aufl. 1985

Prisma. Modern svensk-tysk ordbok. Stockholm 1. Aufl. 1974, 2. umgearbeitete Aufl. 1980

SAOB $=$ Ordbok öfver svenska språket utgifven af Svenska akademien. Lund 1898ff.

Wahrman, S. N. (1814), Svenskt och Tyskt-Franskt-Engelskt Hand-Lexicon. Örebro

Wikforss, Jonas (1804), Tyskt och Svenskt Lexikon. Stockholm

\section{Sonstige zitierte Literatur}

Buhlmann, Rosemarie (1989), 'Fachsprache Wirtschaft' — gibt es die? In: Jahrbuch Deutsch als Fremdsprache 15, S. 82-108

Jumpelt, R. W. (1954), Mehrsprachige Spezialwörterbücher. Moderne Herstellungsmethoden nach Vorschlägen der UNESCO. In: Nachrichten für Dokumentation 5, S. 111-114 und 179-183; 6 (1955), S. 25-28 und 49-52

Kromann, H.-P./Riiber, T./Rosbach, P. (1984), Überlegungen zu Grundfragen der zweisprachigen Lexikographie. In: Studien zur neuhochdeutschen Lexikographie, Bd. 5 (= Germanistische Linguistik 3-6/84). Hildesheim, S. 159-238

Kromann, H.-P./Thomsen, K. Troels (1989), Akzente der Fachsprachenforschung von heute und morgen. Bericht vom Kopenhagener Werkstattgespräch 1.-2. Juni 1988. In: Terminologie et traduction 1, S. 137-160

Lehnstrup, Rita (1989a), Ordbøger — “de er så mange slagse”. In: SPRINT. Sproginstitutternes tidsskrift Handelshфjskolen i Kфbenhavn 2, S. 13-20 
Neubert, Gunter (1970), Die Darstellung des technischen Wortguts in englisch-deutschen Fachwörterbüchern in der Sicht des Technikers. In: Wiss. Zs. der Technischen Universität Dresden 19, 1970, S. 339-342

Neubert, Gunter (1990), Fachlexikographie — Probleme, Aufgaben, Konzepte. In: Hermes. Tidsskrift for sprogforskning 4, S. 67-83

Rossenbeck, Klaus (1975), "En rak vänster” — 'ein gerader Linker'? Bemerkungen zu einem neuen schwedisch-deutschen Wörterbuch. — "Zwei durch acht macht vier". Weiteres zum schwedisch-deutschen Wörterbuch des Prisma-Verlages. In: Moderna språk 69 , S. 21-33 und 316-333

Rossenbeck, Klaus (1977), "Berått mod” — “berauschter Sinn”? Bemerkungen zum Fachwörterbuch für Recht und Wirtschaft. Schwedisch/Deutsch — Deutsch/Schwedisch von G. Parsenow. Köln 1975. In: Moderna språk 71, S. 77-86

Rossenbeck, Klaus (1980), Fachsprachliche Lexik als Problem der Fremdsprachendidaktik (Schwedisch > Deutsch). In: Moderna språk 74, S. 39-50

Rossenbeck, Klaus (1981), Einige Typen fachsprachlicher Wortbildung im Schwedischen und Deutschen. Methodische Probleme ihrer Beschreibung unter dem Gesichtswinkel der einsprachigen und zweisprachigen Lexikographie. In: Fachsprache. Sonderheft 1: Zweites Europäisches Fachsprachensymposium [...] 1979. o.J. [1981], S. 197-209

Rossenbeck, Klaus (1981a), 'Der *Unfalltüchtige' und 'die *Einundfünfzige' — oder Goddag yxskaft! Zur zweiten Auflage von Modern svensk-tysk ordbok (Stockholm: Bokförlaget Prisma, 1980). In: Germanistisches Bulletin. Mitteilungsblatt schwedischer Germanisten 5, S. 75-103

Rossenbeck, Klaus (1985), Zur gegenwärtigen Lage der schwedisch-deutschen und deutsch-schwedischen Lexikographie. In: Die Brüder Grimm. Erbe und Rezeption (= Acta universitatis stockholmiensis, 32). Hg. A. Stedje. Stockholm, S. 142-154

Rossenbeck, Klaus (1987), Zur Gestaltung zweisprachiger Fachwörterbücher. In: Beads or Bracelet? How do we approach LSP. Selected Papers from the Fifth European Symposium on LSP [1985]. Hg. v. A-m Cornu u.a. o.O., o.J. [1987], S. 274-283

Rossenbeck, Klaus (1989), Rezension zu G. Parsenow, Fachwörterbuch für Recht und Wirtschaft [...], 2. Aufl. 1985. In: Lexicographica 5, S. 227-240

Rossenbeck, Klaus (1989a), Lexikologische und lexikographische Probleme fachsprachlicher Phraseologie aus kontrastiver Sicht. In: Translation and Lexicography (= Paintbrush, 16). Hg. M. Snell-Hornby und E. Pöhl, S. 197-210

Sarcevic', Susan (1988), The challange of legal lexicography: Implications for bilingual

$\checkmark$ ănd multilingual dictionaries. In: ZüriLEX '86 Prodceedings. Papers read at the EURALEX International Congress, University of Zürich, 9-14 September 1986. Hg. M. Snell-Hornby. Tübingen, S. 307-314

Schmidt, W. (1969), Forschungsprobleme im fachsprachlichen Bereich. In: Wiss. Zs. der Technischen Universität Dresden 18, S. 439-446

Wiegand, Herbert Ernst (1988), Was ist eigentlich Fachlexikographie? Mit Hinweisen zum Verhältnis von sprachlichem und enzyklopädischem Wissen. In: Deutscher Wortschatz. Lexikologische Studien. Ludwig Erich Schmitt zum 80. Geburtstag von seinen Marburger Schülern. Hg. H. H. Munske u.a. Berlin, New York, S. 729-790 